\title{
Les Chironomidés (Diptera) du Liban. I. Premier inventaire faunistique.
}

\author{
Z. Moubayed' \\ H. Laville ${ }^{1}$
}

Dans ce travail, les auteurs dressent un premier inventaire faunistique des Chironomidés du Liban ou 142 taxa ont été identifiés dans les deux rivière Oronte et Litani de la plaine de la Bekaa et dans la rivière côtière Beyrouth.

81 espèces se révèlent nouvelles pour la région de la Méditerranée orientale : 131 sont nouvelles pour le Moyen-Orient dont les seules données antérieures concernaient la faune lacustre d'Israël.

La distribution géographique des Chironomidés du Liban permet de situer cette règion au carrefour des influences paléarctique, éthiopienne et orientale.

\section{The chironomids (Dtptera) of Lebanon. I. First faunistic inventory.}

The authors present the first faunistic inventory for Chironomidae of the Lebanon where 142 taxa have been identified from the rivers Oronte and Litani in the Bekaa plain and from a river near Beyrouth.

81 species are new records for the eastern Mediterranean ; 131 are new records for the Middle East, the only previous records being for lake species from Israel.

The geographical distribution of chironomids of the Lebanon indicates that this region can be placed at the cross-roads of palaearctic, ethiopian and oriental influences.

\section{Introduction}

Longtemps la connaissance de la faune des Chironomidés de la Méditerranée orientale est restée limitée à celle d'Israël où une liste de 44 espèces dont 23 d'origine éthiopienne, peut être relevée dans les travaux consacrés aux lacs Tibériade et Houlé (Kugler 1966, Kugler \& Chen 1968, Kugler \& Wool 1968 , Kugler \& Reiss 1973) ou au Bas-Jourdain (Ortal \& Por 1978).

Huit espèces supplémentaires sont signalées de cette région dans la Limnofauna Europaea (Fit tkau

1. Laboratoire d'Hydrobiologie, E.R.A. 702 du C.N.R.S., Université P. Sabatier, 118, route de Narbonne, 31062 Toulouse Cedex.
\& Reiss 1978). Récemment, 63 nouveaux taxa regroupant des récoltes éparses du sud de la Yougoslavie, de Grèce et de l'île de Crête (Reiss 1977, Reiss \& Säwedal 1981), portaient ainsi à 115 le total des espèces (ou taxa) de Chironomidés recensés dans cette partie de la Méditerranée. Dans cet te liste, la prédominance (78\%) des Chironominae traduit une prospection plus intense des milieux stagnants.

Dans le cadre d'une étude écologique de la faune macroinvertébrée de quelques réseaux hydrographiques du Liban, l'un de nous ( $Z$. Moubayed), a récolté des Chironomidés dans la plaine de la Békaa. Cette étude represente le premier inventaire faunistique des Chironomidés du Liban obtenus pour la plupart dans les milieux courants -rivières: Oronte, Litani et Beyrouth-mais également dans des sources résurgences ou marécages avoisinants. Elle comprend, de ce fait, un fort pourcentage $(58,5 \%)$ de représentants de la sous-famille des Orthocladiinae. 


\section{1. - Méthodes et stations étudiées}

Le matériel exuvies nymphales, nymphes, imagos noyés-a été obtenu à l'aide de filets (vide de maille : $240 \mu$ ), soit par dérive dans les milieux courant, soit par récolte dans des zones d'accumulation des milieux calmes (résurgences, marais, méandres...).

De plus, des chasses en vol et à la lumière ont permis la capture d'un important materiel imaginal dans les différentes stations.

Les campagnes de récoltes se sont échelonnées sur trois années : juillet, août, septembre 1980 ; septembre, octobre 1981 ; mars, avril, mai 1982 (elles coïncident avec les périodes d'émergence maximale des imagos : mars à octobre).

La quasi-totalité des stations prospectées (stations 1 à 11) est située dans la plaine de la Békaa, orientée N.O.-S.O. à $1000 \mathrm{~m}$ d'altitude moyenne. Cette plaine s'étend dans le système karstique du Mont Liban à l'Ouest et de l'Anti-Liban à l'Est (fig. 1).

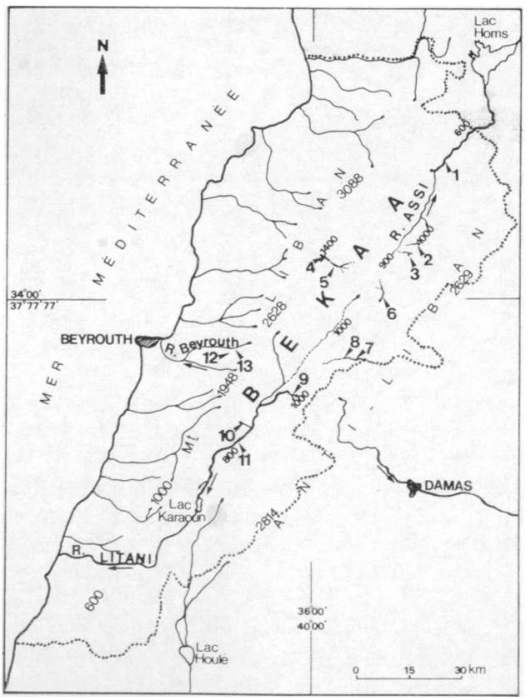

Fig. 1. Liban : localisation des stations étudiées.
Les stations se répartissent dans deux bassins principaux : le bassin de l'Oronte (ou Assi) au NordEst et le bassin du Litani au Sud-Ouest. Deux stations ont également été échantillonnées dans la rivière côtière de Beyrouth. Parfois, plusieurs biotopes différents ont été prospectés à une mème station.

- Les stations 1 à 6 sont situées sur la portion libanaise de la rivière Oronte, de la source $(1400 \mathrm{~m})$ à la frontière Syrienne $(600 \mathrm{~m})$. Cette section, longue de $46 \mathrm{~km}$, correspond à un rhithral ; la rivière a un régime nival: le débit annuel moyen à la station 1 est de $13,6 \mathrm{~m}^{3} / \mathrm{s}$. La station 6 (Baalbek) est localisée dans la zone de partage des eaux : elle appartient en fait aux deux bassins précédents.

- Les stations 7 à 11 , échelonnées entre $1200 \mathrm{~m}$ et $800 \mathrm{~m}$ sont situées sur la rivière Litani depuis la source jusqu'au Lac Karaoun, soit un cours de 100 $\mathrm{km}$ environ. Cette section, à pente faible, peut être considérée comme un épipotamal : elle présente un régime pluvial : le débit annuel moyen à la station 11 est de $9,3 \mathrm{~m}^{3} / \mathrm{s}$.

-. Les stations 12 et 13 ont été prospectées dans le bassin de la rivière côtière de Beyrouth, respectivement à $1000 \mathrm{~m}$ et $700 \mathrm{~m}$ sur le versant ouest du Mont Liban. C'est un cours d'eau à régime pluvial avec un débit annuel moyen de $3.2 \mathrm{~m}^{3} / \mathrm{s}$ (débit maxima : $9,8 \mathrm{~m}^{3} / \mathrm{s}$, débit minima : $0,8 \mathrm{~m}^{3 /} / \mathrm{s}$ ).

Caractéristiques des stations

Pour chaque station nous récapitulons:

- le nom de la localité la plus proche ;

- l'altitude :

- le type de milieu;

- la présence ou l'absence de ripisylve ;

- le substrat:

- la végétation ;

- les températures (minima, maxima);

- les caracteristiques principales des différents biotopes.

\section{1. - Bassin de l'Oronte}

- Station 1: Hermel, $650 \mathrm{~m}$.

Station à l'aval de la source Zarka, source principale de l'Oronte. Rhithral à ripisylve avec deux types de biotopes. $\mathrm{T}^{\circ} 10-15^{\circ} \mathrm{C}$.

1 a : courant rapide avec blocs couverts ou non de bryophytes; 
$1 \mathrm{~b}$ : méandres à courant lent avec substrat de sable et de macrophytes rivulaires.

- Station 2 : Elaïn, $950 \mathrm{~m}$.

Source karstique à faible débit, substrat de cailloux et graviers sans végétation. $T^{\circ} 9-12^{\circ} \mathrm{C}$.

- Station 3 : Labwé, $1000 \mathrm{~m}$.

Résurgences karstiques canalisées à débit moyen ; courant modéré à lent ; substrat grossier : bryophytes peu denses sur les rives. $\mathrm{T}^{\circ} 9.12^{\circ} \mathrm{C}$.

- Station 4 : Yammouné, $1400 \mathrm{~m}-1300 \mathrm{~m}$.

$4 \mathrm{a}$ : source rhéocrène intermittente à courant très rapide : bryophytes sur rochers. $T^{\circ} 8,9-9,5^{\circ} \mathrm{C}$.

$4 \mathrm{~b}$ : résurgences karstiques à courant lent ; macrophytes. $\mathrm{T}^{\circ} 8.10^{\circ} \mathrm{C}$.

$4 c$ : réunion des sources en un cours d'eau avec méandres : subst rat de cailloux et graviers avec des macrophytes.

$4 d$ : petit lac temporaire : substrat de graviers et de sable limoneux; quelques macrophytes sur les bords. $\mathrm{T}^{\circ} 8,5 \cdot 16^{\circ} \mathrm{C}$. Les eaux s'infiltrent en profondeur à travers des " ponors " ou "bétoires " pour réapparaître $41 \mathrm{~km}$ plus loin à la source principale Zarka (niveau station 1).

- Station 5 : Chlifa, 1200-1100 M.

Rhitral formé par les eaux de captage de Yammouné (St. 4). $\mathrm{T}^{\circ} 8,5-13^{\circ} \mathrm{C}$.

5a: courant très rapide; tapis très dense de bryophytes entrecoupé de mouilles; substrat rocheux.

5b; courant lent; substrat fin avec macrophytes.

- Station 6 : Baalbek, $1150 \mathrm{~m}$.

6 : résurgence karstique à courant lent ; substrat fin avec macrophytes. $T^{\circ} 9-12,5^{\circ} \mathrm{C}$.

6b: aval de la résurgence : rhitral à courant modéré ; substrat varié à bryophytes et macrophytes peu denses. $\mathrm{T}^{\circ} 9-16^{\circ} \mathrm{C}$.

\section{2. - Bassin du Litant.}

- Station 7 : affluent Yahfoufa du Litani, $1200 \mathrm{~m}$. Cours supérieur: ripisylve : courant modéré avec substrat de blocs recouverts de bryophytes. $\mathrm{T}^{\circ}$ $11-14^{\circ} \mathrm{C}$.

- Station 8 : Janta, affluent Yahfoufa.

8a : cours inférieur, $1100 \mathrm{~m}$; ripisylve; courant modéré; substrat de blocs recouverts de bryophytes ou d'algues. $\mathbf{T}^{\circ} 12-14^{\circ} \mathrm{C}$.

$8 \mathrm{~b}$ : mare dans le voisinage du Yahfoufa ; substrat vaseux avec algues filamenteuses et macrophytes.

- Station 9 : Anjar-Chamsine, affluent Ghozayel du Litani, $1000-900 \mathrm{~m}$.

$9 \mathrm{a}$ : résurgences karstiques à substrats variés : cailloux en courant modéré et vase en courant lent ou nul. $T^{\circ} 14-18^{\circ} \mathrm{C}$.

$9 \mathrm{~b}$ : épipotamal à courant lent et macrophytes très denses. $\mathrm{T}^{\circ} 13.20^{\circ} \mathrm{C}$.

- Station 10: Ammik, $850 \mathrm{~m}$.

10a : résurgences karstiques, limnocrènes saisonnières avec remous à la sortie des eaux; macrophytes denses. $\mathrm{T}^{\circ} 14-16^{\circ} \mathrm{C}$.

$10 \mathrm{~b}$ : marais et roubines à l'aval des résurgences ; végétation macrophytique très dense et diversifiée. $\mathrm{T}^{\circ} 8.25^{\circ} \mathrm{C}$.

- Station 11 : Jib-Jennine, $800 \mathrm{~m}$.

Cours axial du Litani dépourvu de ripisylve: mésopotamal pollué ; substrat vaseux à macrophy. tes denses et algues filamenteuses. $\mathrm{T}^{\circ} 12-23^{\circ} \mathrm{C}$.

\section{3. - Basșin du Beyrouth.}

- Station 12 : Baalechmay, $1000 \mathrm{~m}$.

Source accessoire de la rivière Beyrouth, à courant très lent et sédiments fins (la source principale Hammana était inaccessible pendant nos campagnes).

- Station 13 : rivière Beyrouth, Hammana : $700 \mathrm{~m}$. Cours d'eau de type méditerranéen avec une ripisylve ; courant rapide à modéré en hiver, lent à très lent en été avec développement de bryophytes en courant et de macrophytes sur les rives. $T^{\circ} 8-27^{\circ} \mathrm{C}$ 


\section{2. - Liste des espèces de Chironomidés du Liban (1980-1982).}

Nous donnons ci-après la liste des 142 taxa identifiés à partir d'imagos mâles (Im), de nymphes (N) ou d'exuvies nymphales $(E)$ avec leurs stations respectives de récoltes. Les espèces précédées d'un astérisque sont nouvelles pour la région de la méditerranée orientale.

\section{Tanypodinae.}

Ablabesmyia longistyla Fitt.

+ Krenopelopia binotata (Wied.)

Macropelopia nebulosa (Mg.)

+ Paramerina cingulata (Walk.)

+P. divisa (Walk.)

P. sp. (Griechenland) Fitt.

Procladius choreus (Mg.)

P. culiciformis (Mg.)

+ Rheopelopia maculipennis (Zett.)

Tanypus punctipennis (Meig.)

+ Thienemannimyia $\mathrm{sp}$.

+ Zavrelimyia berberi Fitt.

\section{Dlamesinae'}

+ Boreopheptagyia legeri (G.)

+Diamesa kasymovi Kown. \& Kown.

+D. modesta Ser. Tos.

+D. sakartvella Kown. \& Kown.

+Potthastia gaedii ( $M g$.)

Prodiamesa olivacea (Mg.)

+Pseudodiamesa nivosa (G.)

Ps. sp.

+Sympotthastia zavreli Pag.

\section{Orthocladinae}

Brillia longifurca $\mathrm{K}$.

B. sp $1 \mathrm{gr}$. flavifrons

B. $\mathrm{sp} 2 \mathrm{gr}$. flavifrons

+ Camptocladius stercorarius (d. Geer)

+ Cardiocladius capucinus (Zett.)

Chaetocladius melaleucus ( $\mathbf{M g}$.)

+ C. perennis (Mg.)

+ Corynoneura celtica Edw.

C. lobata Edw.

C. scutellata Winn.
Im, $E$ (11)

IM, N (5 b)

E (5b/6a/13)

$\operatorname{Im}, \mathrm{E}(6 \mathrm{a} / 9 \mathrm{~b} / 11 / 12 / 13)$

$\operatorname{Im}, E(9 b / 11 / 12 / 13)$

E (1b)

$\operatorname{Im}, \mathrm{N}(4 \mathrm{~d} / 9 \mathrm{~B} / 10 \mathrm{~b} / 11 / 12 / 13)$

Im, N $(9 \mathrm{~b} / 10 \mathrm{~b} / 11)$

E (1b)

$\operatorname{Im}(11)$

$\mathrm{E}$ (13)

$\operatorname{Im}(4 b / 12)$
Im, N, E (1a/3/4ac/5a/7/13)

Im, N, E (4ac/5a/13)

Im, N, E (4a)

$\operatorname{Im}, \mathbf{N}, \mathrm{E}(4 \mathrm{a})$

$\operatorname{Im}, E(1 \mathrm{a} / 13)$

$\operatorname{Im}, \mathrm{N}, \mathrm{E}(4 \mathrm{bcd} / 5 \mathrm{~b} / 6 \mathrm{a} / 10 \mathrm{a} / 11 / 13)$

Im, N, E (4ac)

$\mathrm{N}, \mathrm{E}(4 \mathrm{c})$

Im, N, E (11)
$\operatorname{Im}, \mathrm{E}(1 \mathrm{ab} / 9 \mathrm{a} / 11 / 13)$

Im (9a)

$\operatorname{Im}(9 a)$

Im (7)

Im, N, E (1ab/3/4cd/5b/6/7/8a/13)

Im, N, E (4bcd/6a/10a)

$\operatorname{Im}(4 b d / 5 b / 9 a)$

Im, N, E (1ab/5b/9a)

$\operatorname{Im}, \mathrm{N}, \mathrm{E}(2 / 3 / 4 \mathrm{bc} / 5 \mathrm{ab} / 6 \mathrm{ab} / 7 / 8 \mathrm{a} / 9 \mathrm{a} / 13)$

Im, N, E (1b/4bc/5b/9/10/11)

1. Nous remercions $\mathbf{M}$. B. Serra-Tosio (Grenoble) d'avoir bien voulu identifier les Diamesinae appartenant aux genres Boreoheptagyia, Diamesa et Pseudodiamesa. 
+ Cricotopus annulator $\mathbf{G}$.

C. bicinctus (Mg.)

C. guttatus Hirs.

+ C. similis G.

C. sylvestris (Fabr.)

C. trifascia Edw.

C. trifasciatus (Mg.)

C. vierriensis $\mathbf{G}$.

C. sp.

Eukiefferiella claripennis (Lundb.)

+ E. coerulescens $(K$.)

+E. cyanea Th.

+ E. devonica (Edw.)

+ E. dittmari Lehm.

+ E. fittkaui Lehm.

+ E. fuldensis Lehm.

+ E. ilklevensis (Edw.)

E. lobifera $G$.

+ E. minor (Verr.)

+ E. pseudumontana $G$.

+ Heleniella ornaticollis (Edw.)

+ Hydrobaenus? rufus (K.)

H. sp.

+Limnophyes gurcicola Edw.

+L. minimus (Mg.)

L. $\mathrm{spl}$

L. sp2

L. $\mathrm{sp} 3$

L. $\mathrm{sp4}$

+ Metriocnemus gracei Edw.

M. hirticollis (Staeg.)

$M$. hygropetricus $K$.

Nanocladius bicolor (Zett.)

$+N$. rectinervis $(K$.

+ Orthocladius (Eudactylocladius) obtexens $\mathrm{Br}$.

+ O. (Euorthocladius) thienemanni (K.)

+0 . ? (Orthocladius) excavatus Br.

+0 . (O) frigidus (Zett.)

o. (O) saxicola (K.)

O. $\mathrm{sp} 1$

O. sp2

O. $\operatorname{sp} 3$

Paracladius alpicola (Zett.)

P. conversus (Walk.)

+ Paracricotopus niger (K.)

+ Parametriocnemus stylatus $(\mathrm{K}$.

+ Paraphaenocladius impensus (Walk.)

$+P$. irritus (Walk.)

+Pratrichocladius rufiventris (Mg.)
$\operatorname{Im}, \mathbf{E}(1 \mathrm{ab} / 13)$

Im, N, E (6ab/9ab/10a/11)

Im (lab)

Im, E (4bc/7/13)

$\operatorname{Im}, N, E(1 \mathrm{~b} / 4 \mathrm{~cd} / 5 \mathrm{~b} / 6 \mathrm{a} / 9 \mathrm{ab} / 10 \mathrm{ab} / 11)$

Im, N, E (6ab/7/8a/11/13)

Im, E $(6 a / 9 a b / 11)$

$\operatorname{Im}, \mathrm{E}(1 \mathrm{~b} / 11)$

In, N, E (lab?)

Im, E (10a)

Im, N, E (lab/5a/7/8a)

Im, N, E (1a/4a)

Im, E (1 $\mathrm{a} / 7)$

Im, N, E (1ab/4c/5a/6b/7/8a/13)

Im, N, E (4ac)

$\operatorname{Im}, N, E(1 a b / 2 / 3 / 4 c / 5 a / 6 b / 7 / 8 a / 9 a / 10 a / 13)$

Im, E (1a)

Im, E (1a/5a)

Im, N, E (4ac)

Im, $E / 1 a / 5 a / 6 b / 7 / 13)$

Im, N, E (la)

Im, E $(7 / 8 \mathrm{a} / 9 \mathrm{a})$

Im, N, E (9ab/10ab/11)

Im, $N(5 a b)$

$\operatorname{Im}, \mathrm{N}(1 \mathrm{~b} / 2 / 3 / 4 \mathrm{~cd} / 5 \mathrm{~b} / 6 \mathrm{a} / 7 / 8 \mathrm{a} / 9 \mathrm{ab} / 10 \mathrm{ab} / 11 / 12 / 13)$

$\operatorname{Im}(9 \mathrm{ab} / 10 \mathrm{ab} / 11)$

$\operatorname{Im}(3 / 5 b)$

$\operatorname{Im}(4 c d / 5 b / 6 a / 10 a b)$

Im (4bcd)

$\operatorname{Im}(5 a)$

$\operatorname{Im}(4 b / 9 a)$

$\operatorname{Im}(3 / 4 b c d / 5 b / 6 a / 9 a / 10 a)$

Im (1b)

Im, N, E (1b/3/4cd/5b/6a/7/8ab/9ab/10ab/11/13)

Im, N, E (1ab/4bc/5ab/6ab/7/8a/9a/10a/13)

Im, N, E (1ab/2/3/4c/5a/6b/7/8a/9ab/10a/11/13)

Im, E ( $\mathrm{a} a / 3 / 4 b c)$

Im, N, E (1ab/4bc/5a/6b/9a/10a/13)

Im, N, E (lab/4bcd/5b/6a/7/8a/10ab)

$\operatorname{Im}, N, E(1 a b / 4 c)$

Im, N, E (4c)

$\operatorname{Im}, N, E(9 b / 10 a / 11)$

Im, $E(1 b / 4 b d)$

Im, E (4bd/6a)

Im, N, E (7/8a)

Im, E (1ab/13)

$\mathrm{Im}, \mathrm{N}, \mathrm{E}(\mathrm{lab} / 4 \mathrm{bcd} / 5 \mathrm{~b} / 10 \mathrm{ab} / 12 / 13)$

Im, N, E (lab/10ab)

Im, N, E

(1ab/2/3/4bcd/5ab/6ab/7/8a/9ab/10a/11/13)) 
+ Psectrocladius ? barbimanus Edw.

+ P. ? edwardsi $\mathrm{Br}$.

+ Pseudorthocladius curtistylus (G.)

+Pseudosmittia curticosta (Edw.)

$+P$. trilobata (Edw.)

+ Rheocricotopus ? atripes $\mathrm{K}$.

$+R$. chalybeatus ( $E d w$.)

$+R$. effusus (Walk.)

$+R$. fuscipes (K.)

+ R. ? glabricollis (Mg.)

+ Smittia aquatilis (G.)

$+\mathrm{S}$. ? contingens (Walk.)

$+S$. foliacea $(K$.

$+S$. hamata Freem.

$+\mathrm{S}$. leucopogon $(\mathrm{Mg}$.)

+ S. pratorum G.

S. $\mathrm{sp} 1$

S. sp 2

+Thienemannia libanica n. sp.

+ Thienemanniella morosa Edw.

T. vittata Edw.

+ Trichocladius micans $(\mathrm{K}$.

+ Tvetenia bavarica $G$.

$+\mathbf{T}$. calvescens Edw.

\section{Chironominae}

\section{Chironomini}

+Chironomus aprilinus Meig.

+C. bernensis Wülk \& Klöt

C. dorsalis (Mg.)

C. nuditarsis Keyl.

C. riparius (Mg.)

+Cryptochironomus rostratus $\mathrm{K}$.

Dicrotendipes pilosimanus $\mathrm{K}$.

+Glyptotendipes? gripekoveni K.

+ Harnischia fuscimana (K.)

+ Kiefferulus tendipediformis G. Leptochironomus stilifer Freem. Microtendipes pedellus (de Geer)

+ Parachironomus parilis (Walk.)

+ Paracladopelma camptolabis $\mathbf{k}$.

P. sp. gr. nigritula

+ Paratendipes albimanus (Meg.)

P. nudisquama Edw.

Pentapedilum sp.

Phaenopsectra flavipes (Mg.)

Polypedilum cultellatum G.

P. scalaenum Schr.
Im (10a)

$\operatorname{Im}(4 \mathrm{~cd} / 10 \mathrm{a})$

$\operatorname{Im}(1 \mathrm{ab} / 3)$

$\operatorname{Im}(3)$

$\operatorname{Im}(3 / 4 c d / 5 b / 10 a b / 11)$

$\operatorname{Im}(3 / 12 / 13)$

Im, N, E (1ab/3/7/8a/13)

$\operatorname{Im}, \mathrm{E}(9 \mathrm{ab} / 10 \mathrm{a} / 11)$

Im, N, E (lab/4bcd/5b/6ab/7/8a/9ab/10a/11)

$\operatorname{Im}(7)$

Im (5b)

Im (4bd)

Im (4bd)

$\operatorname{Im}(1 b / 9 b)$

$\operatorname{Im}(\mathrm{lb})$

$\operatorname{Im}(9 a b / 10 a / 11 / 12 / 13)$

Im (4b)

$\operatorname{Im}(4 \mathrm{~cd} / 11)$

Im, N, E (5a)

Im, N, E (1a)

Im, N, E (4c/5b/6a/9ab/10ab/11/12/13)

$\operatorname{Im}(9 \mathrm{~b} / 13)$

Im, N, E (4bc/5a)

Im, N, E (4c/5a/7/8a/13)

Im, N, E (2/3/4d/5b/8b/10ab/11/12/13)

$\operatorname{Im}(9 \mathrm{~b} / 11)$

$\operatorname{Im}(4 \mathrm{~d})$

Im (10a)

$\operatorname{Im}(10 \mathrm{~b} / 11)$

E (9a)

Im, N, E (1b/6a/9ab/10ab/11)

$\operatorname{Im}(4 d)$

Im, N, E (3/9ab/10ab/11/13)

E (1b)

$\operatorname{Im}(11)$

$\operatorname{Im}, E(1 b / 6 a / 9 a / 10 a / 13)$

$\operatorname{Im}(10 \mathrm{a})$

Im, N, E ( $1 a b / 4 c d / 9 a)$

Im, N, E (4cd/5b/13)

Im, $E$ ( $1 \mathrm{ab})$

$\operatorname{lm}, \mathrm{E}(1 \mathrm{~b} / 2)$

$\operatorname{Im}(9 \mathrm{~b} / 10 \mathrm{~b} / 1 \mathrm{l})$

Im, E (6a/9ab/10ab/11/13)

$\operatorname{Im}(9 \mathrm{ab} / 11)$

$\operatorname{Im}(7 / 8 \mathbf{a} / 13)$ 
P. sp 1

P. sp 2

P. sp 3

Stictochironomus maculipennis (Mg.)

+S. pictulus (Mg.)

\section{Tanytarsini}

+ Cladotanytarsus atridorsum (K.)

+ C. vanderwulpi Edw.

Micropsectra atrofasciata $\mathbf{K}$.

$+\mathrm{M}$. junci $(\mathrm{Mg}$.)

M. ? lindrothi $\mathrm{G}$.

+ M. ? notescens (Walk.)

Paratanytarsus bituberculatus (Edw.)

$+P$. confusus Pal.

$+P$. inopertus (Walk.)

Rheotanytarsus curtistylus G.

+ Stempellina subglabripennis $\mathrm{Br}$.

Virgatanytarsus hulensis (Kug. \& Reiss)

Les récoltes proviennent essentiellement des deux principaux réseaux hydrographiques du Liban : le bassin de l'Oronte et le bassin du Litani ; le bassin du Beyrouth a été étudié de façon moins intensive. Des milieux différents ont été prospectés : un rhitral à courant rapide (Oronte) et un épipotamal à courant lent (Litani) mais également des résurgences karstiques froides, des sources rhéocrènes et limnocrènes et des zones de marécages.

Le tableau I récapitule la répartition des espèces dans les 13 stations étudiées. Sur les 142 taxa différenciés, 121 sont identifiés au niveau spécifique. Quelques uns des 21 taxas déterminés seulement au niveau générique sont, sans doute, nouveaux pour la science, ce que devrait confirmer leur étude taxonomique ultérieure.

La répartition des espèces dans les principales
$\operatorname{Im}, \mathrm{N}, \mathrm{E}(1 \mathrm{ab} / 5 \mathrm{~b} / 6 \mathrm{a} / 7 / 8 \mathrm{a})$

Im, N, E (1b/4bd/5b/7)

$\operatorname{Im}(9 b)$

$\operatorname{Im}, \mathrm{E}(4 \mathrm{~cd} / 6 \mathrm{ab} / 7 / 8 \mathrm{a} / 9 \mathrm{ab} / 10 \mathrm{a} / 11)$

$1 \mathrm{~m}, \mathrm{E}(13)$

Im, N, E (6a)

Im, N, E (6a)

$\operatorname{Im}, \mathrm{N}, \mathrm{E}(1 \mathrm{~b} / 2 / 3 / 4 \mathrm{bcd} / 5 \mathrm{~b} / 6 \mathrm{a} / 7 / 8 \mathrm{a} / 10 \mathrm{a} / 11 / 13)$

Im, N, E $(4 \mathrm{bc} / 5 \mathrm{~b} / 6 \mathrm{a} / 13)$

$\mathrm{Im}, \mathrm{E}(4 \mathrm{bc} / 5 \mathrm{~b} / 10 \mathrm{a})$

Im, N, E (4bcd/5b)

E (13)

Im, E $(9 \mathrm{ab} / 10 \mathrm{a} / 11)$

Im, E (10ab/11)

Im, N, E ( $1 \mathrm{ab} / 3 / 6 \mathrm{a} / 7 / 8 \mathrm{a} / 9 \mathrm{~b})$

Im, N, E (9a)

Im (9b)

sous-familles ou tribus et leur fréquence respective sont données, pour chacun des trois bassins, dans le tableau II.

Avec un total de 108 espèces, dont 42 exclusives, le bassin de l'Oronte apparaît plus diversifé que celui du Litani où 90 espèces, dont 28 exclusives, ont été identifiées. 43 espèces, dont 3 exclusives, sont recensées dans la rivière Beyrouth prospectée, il est vrai, de façon moins suivie; 58 espèces sont communes aux bassins de l'Oronte et du Litani.

Sur le tableau II, oủ sont récapitulées la répartition et la fréquence des espèces regroupées par sousfamilles ou tribus dans les trois rivières étudiées, nous pouvons voir que les Orthocladiinae et les Diamesinae représentent ensemble $65 \%$ des récoltes totales pour $26,8 \%$ de Chirominae (Chironomini + Tanytarsini).

Tableau I. Répartition dẹ espèces de Chironomides recensées dans chacune des 13 stations et regroupées par sous-familles ou tribus.

\begin{tabular}{|c|c|c|c|c|c|c|c|c|c|c|c|c|c|}
\hline STATIONS & 1 & 2 & 3 & 4 & 5 & 6 & 7 & 8 & 9 & 10 & 11 & $12+13$ & Total \\
\hline Tanypodinae & 2 & & & 2 & 2 & 2 & & & 4 & 2 & 6 & 6 & 12 \\
\hline Diamesinae & 2 & & 1 & 7 & 3 & $i$ & I & & & 1 & 2 & 4 & 9 \\
\hline Orthocladiinae & 38 & 5 & 16 & 39 & 31 & 22 & 22 & 17 & 28 & 28 & 20 & 23 & 83 \\
\hline Chironomini & 8 & 2 & 2 & 7 & 4 & 6 & 4 & 4 & 11 & 10 & 10 & 7 & 26 \\
\hline Tanytarsini & 2 & 1 & 2 & 4 & 4 & 5 & 2 & 2 & 4 & 4 & 3 & 3 & 12 \\
\hline TOTAL & 52 & 8 & 21 & 59 & 44 & 36 & 29 & 23 & 47 & 45 & 41 & 43 & 142 \\
\hline
\end{tabular}


Tableau II. Répartion et fréquence des espèces de Chironomides regroupées par sous-familles ou Iribus dans les trois rivières étudiées : l'Oronte, le Litani, le Beyrouth.

\begin{tabular}{|c|c|c|c|c|c|c|c|c|}
\hline & \multicolumn{2}{|c|}{ ORONTE } & \multicolumn{2}{|c|}{ LITANI } & \multicolumn{2}{|c|}{ BEYROUTH } & \multicolumn{2}{|c|}{ TOTAL } \\
\hline & $N$ & $\%$ & $\mathbf{N}$ & $\%$ & $N$ & $\%$ & $\mathrm{~N}$ & $\%$ \\
\hline Tanypodinae & 7 & 6,5 & 6 & 6,7 & 6 & 14 & 12 & 8,4 \\
\hline Diamesinae & 8 & 7.4 & 3 & 3,3 & 4 & 9,3 & 9 & 6,3 \\
\hline Orthocladiinae & 70 & 64,8 & 52 & 57,8 & 23 & 53,5 & 83 & 58,5 \\
\hline Chironomini & 15 & 13,9 & 20 & 22,2 & 6 & 14 & 26 & 18,3 \\
\hline Tanytarsini & 8 & 7,4 & 9 & 10 & 4 & 9,3 & 12 & 8,4 \\
\hline TOTAL & 108 & & 90 & & 43 & & 142 & \\
\hline
\end{tabular}

\section{3. - Analyse faunistique et biogéographique}

\section{1. - Tanypodinae.}

9 des 12 espèces de Tanypodinae identifiées sont largement répandues en Europe. 2 espèces, par contre, Paramerina sp. (Griechenland) et Zavrelimyia berberi présentent une répartition méditerranéenne : la première, récoltee à l'état d'exuvie dans l'Oronte (St. lb), n'est signalée qu'en Grèce et en Espagne, dans les Picos de Europa (Fit tkau 1962); la deuxième, $Z$. berberi récoltée à l'état d'imago mâle dans deux stations (4b et 12), se différencie des espèces européennes par un rapport antennaire inférieur à 2 (A.R. $=1,65$ ); elle n'était connue que d'un lac marocain du haut Atlas situé à $2800 \mathrm{~m}$ d'altitude (Fittkau 1962).

\section{2. - Diamesinae}

La majorité des Diamesinae proviennent du bassin de l'Oronte ( 8 espèces) toujours au-dessus de $1000 \mathrm{~m}$ d'altitude ; elles sont plus rares dans le Litani (3 espèces) et le Beyrouth (4 espèces). Deux espèces, Diamesa kasymovi et $D$. sakartvella, sont uniquement connues du Caucase (Kownacki \& Kownacka 1973), une troisième, $D$. modesta, est signalée à la fois du Caucase (Kownacki \& Kownacka 1974) et des
Alpes (Serra-Tosio 1973). la distribution géographique de ces espèces orophiles souligne la plus grande affinité de la faune de la rivière Oronte avec celle du Caucase qu'avec celle des montagnes occidentales. De plus, la présence de ces espèces au Liban confirme l'hypothèse émise par plusieurs auteurs (Serra-Tosio 1973, Kownacki \& Kownacka 1974) selon laquelle la voie d'acheminement empruntée par les Diamesini d'origine africaine se situerait à l'Est de la Méditerranée. Potthastia gaedii et Sympotthastia zavreli ont été récoltées à plus basse altitude $(600$ à $800 \mathrm{~m})$. Une Pseudodiamesa sp. capturée dans le cours d'eau de Yammouné (4c) est probablement nouvelle.

\section{3. - Orthocladilnae}

Avec 83 taxa, c'est la sous-famille la mieux représentée surtout dans le bassin de l'Oronte (70 taxa) où les stations prospectées appartiennent essentiellement à un rhithral.

Parmi les 11 espèces rhéophiles appartenant au genre Eukiefferiella, 3 (E. cyanea, E. fittkaui et $E$. minor) sont cantonnées, tout comme certaines Diamesinae, dans les sources et en ava! des résurgences sténothermes d'eau froide qui constituent pour ces espèces des habitats refuges d'un grand intérêt écologique.

La plujpart des especes identifiées sont largement répandues en Europe. Deux espèces sont connues de la Méditerranée orientale : Cricotopus guttatus, 
signalée de Grèce et d'Israêl et Thienemannia liba. nica $\mathrm{n}$. sp., espèce nouvelle dont l'imago mâle et la nymphe seront decrits prochainement. Deux Brillia sp1 et sp2 du groupe flavifrons (Oliver \& Roussel) 1983), récoltées dans les résurgences karstiques du Litani (Anjar, St. 9) sont sûrement nouvelles pour la science, leur étude taxonomique est en cours. Deux orthocladiinae Smittia hamata et Trichocladius micans n'étajent signalées que de la région éthiopienne : la première du Transvaal, la seconde d'Afrique du Sud, du Zaire, de l'Ouganda (lac Victoria), d'Ethiopie et du Yemen (Freeman 1956).

\section{4. - Chironominae}

Sur les 38 taxa de cette sous-famille, 26 appartiennent à la tribu des Chironomini, 12 à celle des Tanytarsini. Leurs récoltes proviennent surtout de l'épipotamal du Litani (26 taxa).

A côté d'espèces assez largement répandues en Europe, 2 espèces sont à la fois connues de la région éthiopienne et d'Israël : Leptochironomus stilifer et Virgatanytarsus hulensis ${ }^{\text {(Freeman 1957, Kugler }}$

1. Le genre Virgaranyarsus a été récemment créé par Pinder (1982) pour les 6 especes du groupe a triangularis o de Reiss et Fittkau ([971).
\& Reiss 1973). Une troisième espèce, Dicrotendipes pilosimanus, présente une distribution intéressante puisqu'elle est signalée de la région méditerranéenne (Israël, Grèce, Maroc) mais également des régions orientale (Inde) et australe (Fittkau \& Reiss 1978) ; Reiss (1977) la considère comme un élément à vaste répartition " panpaléotropicale n.

\section{4. - Conclusion}

Ce travail constitue la première étude faunistique importante consacree aux Chironomidés des eaux courantes de la Méditerranée orientale qui, selon de Lattin (1967), englobe les Balkans, la Turquie, le Moyen-Orient et la Cyrénaique d'Afrique. 81 des 142 taxa identifiés sont nouveaux pour cette région, ce qui porte à 196 le total des especes (ou taxa) actucllement connus dans l'Est-Méditerranée (Tableau III).

Le peuplement des Chironomidés des deux prin. cipales rivières du Liban est composé d'un fort pourcentage d'espèces paléarctiques largement répandues en Europe. Dans ce peuplement nous avons mis en évidence un petit noyau d'éléments paléarctiques circum-méditerranécns, Cricotopus guttatus, Paramerina sp. (Griechenland), Zavrelimyia berberi. Thienemannia libanica n. sp. et ajouté deux Orthocladiinae. Trichocladius micans et Smittia hamata, à la

Tableau III. Total des Chironomides de la Méditerranée orientale comprenant les espéces maintenant recensées en Israël et dans les Balkans du Sud-Ouest (Reiss 1977) et celles nouvellement citées au Liban : répartition des espèces d'origine éthiopienne actuellement connues dans l'Est Méditerranée.

\begin{tabular}{|c|c|c|c|c|c|c|c|}
\hline & & 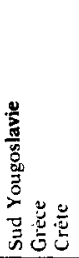 & $\underset{b}{\stackrel{2}{0}}$ & \multicolumn{4}{|c|}{ EST MEDITERRANEE } \\
\hline Tanypodinae & 8 & 10 & 18 & 6 & 24 & 12,2 & 1 \\
\hline Diamesinae & & 3 & 3 & 7 & 10 & 5,1 & \\
\hline Orthocladiinae & 8 & 6 & {$[4$} & 51 & 65 & 33,2 & 2 \\
\hline Chironomini & 30 & 30 & 60 & 10 & 70 & 35.7 & 18 \\
\hline
\end{tabular}


liste des 23 espèces éthiopiennes (dont 18 Chironomini) déjà connues de la Méditerranée orientale. Ces espèces d'origine africaine, qui représentent maintenant $12,8 \%$ du total des Chironomides de l'EstMéditerranée et $14,5 \%$ de la faune chironomidienne du Moyen-Orient, témoignent de l'utilisation de la vallée du $\mathrm{Ni}$ ] comme voie privilégiée de pénétration vers le Nord (Reiss 1977).

La faune des rivières Oronte et Beyrouth issues de la chaine du Mont-Liban présente des affinités avec celle du Caucase comme le montre la présence de Diamesinae orophiles communes, notamment Diamesa kasymovi et $D$. sakartvella, qui paraissent ici à la limite méridionale de leur aire de répartition.

D'après les données de la littérature sur Israël et ce travail sur le Liban, 172 espèces (ou taxa) sont maintenant recensés au Moyen-Orient, 52 en Israël et 142 au Liban.

Parmi les espèces à l'étude, certaines, à l'exem. ple de Thienemannia libanica $\mathrm{n}$. sp., se révèleront sans doute nouvelles; elles pourraient servir à caractériser cette région du Moyen-Orient en compagnie des 2 especes levantines actuellement indigènes d'Israë1, Chaetocladius palestinae G. et Polypedilum tiberiadis $\mathrm{K}$.

\section{Travaux cités}

De Lattin (G.). 1967. - Grundriss der Zoogeographie. G. Fischer, Siutgart.

Fittkau (E.J.). 1962. - Die Tanypodinae (Diptera, Chironomidae). Abh. Larval-syst. Insekten, 6: 1-453.
Fittkau (E.J.) \& Reiss (F.). 1978. - Chironominae. In Illies J., ed Limnofauna Europaea: 404440. G. Fischer, Stuttgart.

Freeman (P.). 1956. - A siudy of the Chironomidae (Diptera) of Af rica South of the Sahara. II. Bull. Br. Mus. nat. Hist., Enr. $4: 285-368$.

Freeman (P.). 1957. - A study of the Chironomidae (Diptera) of Africa South of the Sahara. III. Bull. Br. Mus. nat. Hist., Ent., $5: 321-426+1 \mathrm{pl}$.

Kownacki (A.) \& Kownacka (M.). 1973. - Chironomidae (Diptera) from the Caucasus. Diameso Waltl group steinboecki. Bul. Acad. Pol. Sc., Sér. Sc. biol., II, 21 (1): $27,37$.

Kownacki (A.) \& Kownacka (M.). 1974. - Relation of Chironomi. dae from Tatra and the Caucasus Mis. Ent tidskr., 95, suppl. : 129.138.

Kugler (J.). 1966. - Vorläufige Mitteilung über die Chironomidenfaun des Tiberiassees. Gewäss. Abwäss., 41/42: 70-84.

Kugler (J.) 1971. - The developmental stages of Leptochironomus stilifer (Diptera. Chironomidae) and the characters of the genus Leptochironomus. Can. Ent., $103: 341-346$.

Kugler (J.) \& Chen (H.). 1968. - The distribution of Chironomid larvae in Lake Tiberias (Kinneret) and their occurence in the food fish of the lake. Israël J. Zool, $17: 97-115$.

Kugler (J.) \& Reiss $\{F$.$\} . 1973. - Die rriangularis-Gruppe der Gat-$ tun Tanytarsus v.d.W. (Chironomidae, Diptera). Ent. Tidskr., $94: 59-82$.

Kugler (J.) \& Wool (D.). 1968. - Chironomidae (Diptera) from the Hula Nature Preserve, Israell. Ann. zool. fenn, 5 : 76-83.

Oliver (D.R.) \& Roussel (M.E.). 1983. - Redescription of Brillia Kieffer (Diptera : Chironomidae) with descriptions of nearctic species. Can. Ent., 115: 257-279.

Orial (R.) \& Por (F.D.). 1978. - Effect of hydrological changes on aquatic communities in the Lower Jordan River. Verh. Intermat. Verein. Limnol, 20 : 1543-1551.

Pinder (L.C.V.). 1982. Virgatanytarsus new genus for the "triangularis " group of the genus Tanytarsus van der Wulp. Spixiana, 5 (1) : $31-34$.

Reiss (F). 1977, - Verbreitungsmuster bei paläarktischen Chironomidearten (Diptera, Chironomidae). Spixiana, 1 : 85-97.

Reiss (F.) \& Fittkau (E.J.). 1971. - Taxonomie und Okologie europäisch verbreiteter Tanytarsus-Arten (Chironomidae, Diptera). Arch. Hydrobiol., Suppl. 40:75-200.

Reiss (F.) \& Sawedal (L.). 1981 - Keys to males and pupae of the Paleartic (excl. Japan) paratanytarsus Thienemann \& Bause, 1913, n. cornb., with descriptions of three new species (Diptera: Chironomidae). Ent. Scand, suppl. 15: 73-104

Serra-Tosio (B.). 1973. - Ecologie et biogéographie des Diamesini d'Europe (Diptera, Chironomidae). Trav. Lab. d'Hydrobiol. Pisc. Univ. Grenoble, $63: 5.175$. 